\section{Lattice Oscillations in Crystals}

ThE ions or molecules occupying the cells of a crystal lattice are in general optically anisotropic, and should also be capable of executing small angular oscillations about their positions of equilibrium. If the crystal is illuminated by monochromatic light of frequency $v$, the local fluctuations in optical polarization produced by such oscillations should result in scattered radiations appearing with altered frequency, and theory indicates that these should exhibit peculiar polarization characters.

Taking an individual ion or molecule to be optically an ellipsoid, its polarizability along three mutually perpendicular directions being $A, B, C$ respectively, it is clear that if the light-vector $E$ in the incident beam is parallel to an axis $A$ of the ellipsoid and its angular oscillations are also about the same axis, there would be no fluctuations in optical polarization and therefore no radiations of altered frequency. If, however, the ion or molecule oscillates about a second axis $B$ with an angular amplitude $\theta$ and frequency $v^{*}$ the optical polarization would fluctuate with frequencies $\nu \pm \nu^{*}$; the corresponding scattered radiations would arise from a Hertzian dipole which is parallel to the third axis $C$ of the ellipsoid and has the strength $\frac{1}{2}(C-A) \theta E$, where $\theta=\left(h / 2 \pi^{2} I \nu^{*}\right)$, $I$ is the moment of inertia and $h$ is Planck's constant. The scattered radiations would thus be of maximum intensity in the plane containing the oscillation axis and the incident light-vector and would vanish in the direction perpendicular to this plane. In other words, the displaced lines in the spectrum due to these oscillations in the lattice would disappear in just the direction in which the displaced lines arising from the symmetric internal vibrations of the ion or molecule would be of maximum intensity.

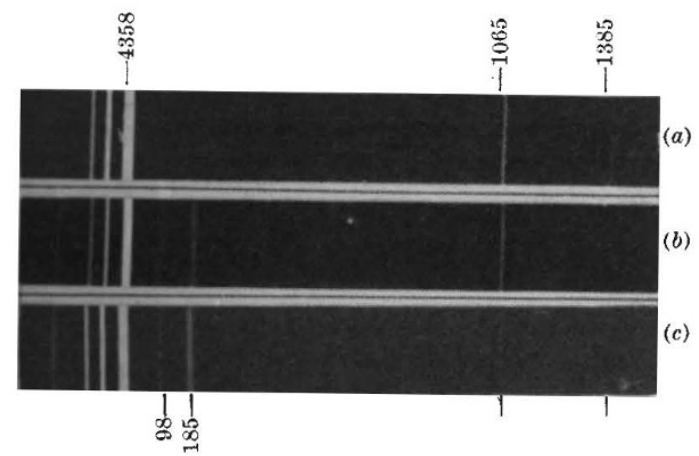

EFFECT OF ORIENTATION OF CRYSTAL ON LIGHT SCATTERING IN SODIUM NITRATE.

$(a),(b)$, and $(c)$ illustrate the foregoing remarks ; they are spectra of the $4358 \mathrm{~A}$. radiation of the mercury arc, transversely scattered by a sodium nitrate crystal, the incident light being plane polarized. The three spectra correspond to three mutually perpendicular settings of the crystal; the directions of the incident light-vector and of observation of the scattered light are normal to each other and remain unaltered throughout. It will be noticed that in $(a)$, in which the optic axis of the crystal is parallel to the direction of observation, the lattice lines $\left(98 \mathrm{~cm}^{-1}\right.$ and $\left.185 \mathrm{~cm} .^{-1}\right)$ have disappeared, while in $(b)$ and $(c)$ they appear strongly. On the other hand, the internal oscillations $\left(1065 \mathrm{~cm}^{-1}\right.$ and $1385 \mathrm{~cm}^{-1}$ ) of the $\mathrm{NO}_{3}$ ion appear strongly in $(a)$ and $(b)$, while in $(c)$ they are very weak. The latter result is to be expected, as (c) corresponds to the case in which the incident light-vector is along the optic axis of the crystal, and the polarizability of the $\mathrm{NO}_{3}$ ions is a minimum in this direction.
C. V. Raman.
Hebbal, Bangalore.
T. M. K. Nedungadi. March 10.

\section{Transmutation of Thorium by Neutrons}

IN a previous letter we have proposed schemes for the disintegration of radioactive noble gases, formed by the bombardment of uranium with slow neutrons ${ }^{1}$. We repeated these experiments, passing the active gases over absorbent carbon instead of through water, which resulted in appreciably higher activities. We were able to confirm our earlier measurements and found 33 minutes as a more accurate value for the longer cæsium-period (instead of 30 minutes). It may be remarked that there is a satisfactory agreement between our results and those obtained by Hahn and Strassmann ${ }^{2}$.

We have now studied the formation of radioactive noble gases by the splitting of thorium nuclei with neutrons in an analogous way. As this process requires fast neutrons, we used the following experimental arrangement. The neutrons were produced by the $(\mathrm{Li}+\mathrm{D})$ reaction. The neutron source was immersed in a concentrated solution of thorium nitrate. Thus, although the water slows down the neutrons, an appreciable part of those present in the liquid still have high energies. A fast stream of nitrogen was passed through the solution and, after being dried with phosphorus pentoxide, entered a $U$-shaped tube filled with active carbon and immersed in liquid nitrogen. The radio elements were extracted from the carbon with water containing cæsium, rubidium, barium, strontium and lanthanum ions. This solution always contains thorium $\mathrm{B}$ and $\mathrm{C}$ originating from thoron absorbed by the active carbon. This fact made it necessary to carry out a lead precipitation before isolating the barium, and to purify as a perchlorate the cæsium contained in the precipitate with antimony trichloride. The irradiations ranged from 5 minutes to 60 minutes.

Except for the 2-minute alkali period, our results were identical with those obtained in the investigation of uranium. We determined the same periods for rubidium (17 min.), barium (87 min.) and cæsium (10 min. and $33 \mathrm{~min}$.). Strontium was found to be inactive. It may be concluded that the splitting of uranium nuclei and of thorium nuclei leads to the same noble gases, and that the schemes suggested for uranium in our first communication hold for thorium too. As the time required for the preparation of the precipitates was longer in the case of thorium than it was in our investigation of uranium, it was impossible to search for the 2-min. alkali period.
A. H. W. Aten, Jun.
C. J. BAKKER.
F. A. Heyn.

Natuurkundig Laboratorium, N. V. Philips' Gloeilampenfabrieken, Eindhoven.
April 5.

${ }^{1}$ Heyn, Aten, jun., and Bakker, Nature, 143, 519 (1939).

2 Hahn and Strassmann, Naturwiz8., 27, 163 (1939). 